Special Issue: 25 years of the Mizar Mathematical Library

FORMALIZED MATHEMATICS

Vol. 22, No. 2, Pages $167-176, \mathbf{2 0 1 4}$

DOI: $10.2478 /$ forma-2014-0017

\title{
Tarski Geometry Axioms
}

\author{
William Richter \\ Departament of Mathematics \\ Nortwestern University \\ Evanston, USA
}

\author{
Adam Grabowski \\ Institute of Informatics \\ University of Białystok \\ Akademicka 2, 15-267 Białystok \\ Poland
}

\author{
Jesse Alama \\ Technical University of Vienna \\ Austria
}

Summary. This is the translation of the Mizar article containing readable Mizar proofs of some axiomatic geometry theorems formulated by the great Polish mathematician Alfred Tarski [8, and we hope to continue this work ${ }^{1}$

The article is an extension and upgrading of the source code written by the first author with the help of miz3 tool; his primary goal was to use proof checkers to help teach rigorous axiomatic geometry in high school using Hilbert's axioms.

This is largely a Mizar port of Julien Narboux's Coq pseudo-code 6]. We partially prove the theorem of [7] that Tarski's (extremely weak!) plane geometry axioms imply Hilbert's axioms. Specifically, we obtain Gupta's amazing proof which implies Hilbert's axiom I1 that two points determine a line.

The primary Mizar coding was heavily influenced by 9] on axioms of incidence geometry. The original development was much improved using Mizar adjectives instead of predicates only, and to use this machinery in full extent, we have to construct some models of Tarski geometry. These are listed in the second section, together with appropriate registrations of clusters. Also models of Tarski's geometry related to real planes were constructed.

MSC: 51A05 51M04 03B35

Keywords: Tarski's geometry axioms; foundations of geometry; incidence geometry

MML identifier: GTARSKI1, version: 8.1.03 5.23.1213

\footnotetext{
${ }^{1}$ The first author ported the code to HOL Light (http://www.cl.cam.ac.uk/ jirh13/hol-light/), which can be found in any recent subversion of HOL Light as hol_light/RichterHilbertAxiomGeometry/TarskiAxiomGeometry_read.ml

(C) 2014 University of Białystok CC-BY-SA License ver. 3.0 or later ISSN 1426-2630(Print), 1898-9934(Online)
} 
The notation and terminology used in this paper have been introduced in the following articles: [2], [3], [5], [1], [1], [10], and [4].

\section{TARski's Geometry Axioms}

We consider Tarski planes which extend 1-sorted structures and are systems

$$
\text { 〈a carrier, a betweenness, an equidistance〉 }
$$

where the carrier is a set, the betweenness is a relation between (the carrier) $\times$ (the carrier) and the carrier, the equidistance is a relation between (the carrier) $\times$ (the carrier) and (the carrier) $\times$ (the carrier)

Let $\mathfrak{S}$ be a Tarski plane.

A point of $\mathfrak{S}$ is an element of $\mathfrak{S}$. Let $A, B, C$ be points of $\mathfrak{S}$. We say that $B$ lies between $A$ and $C$ if and only if

(Def. 1) $\langle\langle A, B\rangle, C\rangle \in$ the betweenness of $\mathfrak{S}$.

Let $A, B, C, D$ be points of $\mathfrak{S}$. We say that $\overline{A B} \cong \overline{C D}$ if and only if

(Def. 2) $\langle\langle A, B\rangle,\langle C, D\rangle\rangle \in$ the equidistance of $\mathfrak{S}$.

Let $A, B, C, X, Y, Z$ be points of $\mathfrak{S}$. We say that $\triangle A B C \cong \triangle X Y Z$ if and only if

(Def. 3) (i) $\overline{A B} \cong \overline{X Y}$, and

(ii) $\overline{A C} \cong \overline{X Z}$, and

(iii) $\overline{B C} \cong \overline{Y Z}$.

Let $A, B, C, D$ be points of $\mathfrak{S}$. We say that $A, B, C, D$ are ordered if and only if

(Def. 4) (i) $B$ lies between $A$ and $C$, and

(ii) $B$ lies between $A$ and $D$, and

(iii) $C$ lies between $A$ and $D$, and

(iv) $C$ lies between $B$ and $D$.

We say that $\mathfrak{S}$ satisfies the axiom of congruence symmetry if and only if

(Def. 5) Let us consider points $A, B$ of $\mathfrak{S}$. Then $\overline{A B} \cong \overline{B A}$.

We say that $\mathfrak{S}$ satisfies the axiom of congruence equivalence relation if and only if

(Def. 6) Let us consider points $A, B, P, Q, R, S$ of $\mathfrak{S}$. Suppose

(i) $\overline{A B} \cong \overline{P Q}$, and

(ii) $\overline{A B} \cong \overline{R S}$.

Then $\overline{P Q} \cong \overline{R S}$.

We say that $\mathfrak{S}$ satisfies the axiom of congruence identity if and only if 
(Def. 7) Let us consider points $A, B, C$ of $\mathfrak{S}$. If $\overline{A B} \cong \overline{C C}$, then $A=B$.

We say that $\mathfrak{S}$ satisfies the axiom of segment construction if and only if

(Def. 8) Let us consider points $A, Q, B, C$ of $\mathfrak{S}$. Then there exists a point $X$ of $\mathfrak{S}$ such that

(i) $A$ lies between $Q$ and $X$, and

(ii) $\overline{A X} \cong \overline{B C}$.

We say that $\mathfrak{S}$ satisfies the axiom of SAS if and only if

(Def. 9) Let us consider points $A, B, C, X, A_{1}, B_{1}, C_{1}, X_{1}$ of $\mathfrak{S}$. Suppose

(i) $A \neq B$, and

(ii) $\triangle A B C \cong \triangle A_{1} B_{1} C_{1}$, and

(iii) $B$ lies between $A$ and $X$, and

(iv) $B_{1}$ lies between $A_{1}$ and $X_{1}$, and

(v) $\overline{B X} \cong \overline{B_{1} X_{1}}$.

Then $\overline{C X} \cong \overline{C_{1} X_{1}}$.

We say that $\mathfrak{S}$ satisfies the axiom of betweenness identity if and only if

(Def. 10) Let us consider points $A, B$ of $\mathfrak{S}$. If $B$ lies between $A$ and $A$, then $A=B$.

We say that $\mathfrak{S}$ satisfies the axiom of Pasch if and only if

(Def. 11) Let us consider points $A, B, P, Q, Z$ of $\mathfrak{S}$. Suppose

(i) $P$ lies between $A$ and $Z$, and

(ii) $Q$ lies between $B$ and $Z$.

Then there exists a point $X$ of $\mathfrak{S}$ such that

(iii) $X$ lies between $P$ and $B$, and

(iv) $X$ lies between $Q$ and $A$.

We say that $\mathfrak{S}$ satisfies seven Tarski's geometry axioms if and only if

(Def. 12) $\mathfrak{S}$ satisfies the axiom of congruence symmetry, the axiom of congruence equivalence relation, the axiom of congruence identity, the axiom of segment construction, the axiom of SAS, the axiom of betweenness identity, and the axiom of Pasch.

\section{Existence Proofs for Tarski Plane}

We consider metric Tarski structures which extend metric structures and Tarski planes and are systems

〈a carrier, a distance, a betweenness, an equidistance〉 
where the carrier is a set, the distance is a function from (the carrier) $\times($ the carrier) into $\mathbb{R}$, the betweenness is a relation between (the carrier) $\times$ (the carrier) and the carrier, the equidistance is a relation between (the carrier) $\times($ the carrier) and (the carrier) $\times($ the carrier $)$.

Let $\mathfrak{M}$ be a metric structure.

A Tarski extension of $\mathfrak{M}$ is a metric Tarski structure and is defined by

(Def. 13) The metric structure of $i t=$ the metric structure of $\mathfrak{M}$.

Let $\mathfrak{M}$ be a non empty metric structure. One can check that every Tarski extension of $\mathfrak{M}$ is non empty.

Let $\mathfrak{M}$ be a non empty reflexive metric structure. Observe that every Tarski extension of $\mathfrak{M}$ is reflexive.

Let $\mathfrak{M}$ be a non empty discernible metric structure. Note that every Tarski extension of $\mathfrak{M}$ is discernible.

Let $\mathfrak{M}$ be a non empty symmetric metric structure. One can verify that every Tarski extension of $\mathfrak{M}$ is symmetric.

Let $\mathfrak{M}$ be a non empty triangle metric structure. Observe that every Tarski extension of $\mathfrak{M}$ is triangle.

Let $\mathfrak{S}$ be a metric structure and $P, Q, R$ be elements of $\mathfrak{S}$. We say that $Q$ is between $P$ and $R$ if and only if

(Def. 14) $\rho(P, R)=\rho(P, Q)+\rho(Q, R)$.

Let $\mathfrak{M}$ be a metric Tarski structure. We say that $\mathfrak{M}$ is naturally generated if and only if

(Def. 15) (i) for every points $A, B, C$ of $\mathfrak{M}, B$ lies between $A$ and $C$ iff $B$ is between $A$ and $C$, and

(ii) for every points $A, B, C, D$ of $\mathfrak{M}, \overline{A B} \cong \overline{C D}$ iff $\rho(A, B)=\rho(C, D)$.

Now we state the proposition:

(1) Let us consider metric structures $\mathfrak{M}, \mathfrak{N}$, elements $X, Y$ of $\mathfrak{M}$, and elements $A, B$ of $\mathfrak{N}$. Suppose

(i) the metric structure of $\mathfrak{M}=$ the metric structure of $\mathfrak{N}$, and

(ii) $X=A$, and

(iii) $Y=B$.

Then $\rho(X, Y)=\rho(A, B)$.

Let $\mathfrak{N}$ be a non empty metric structure. Let us note that there exists a Tarski extension of $\mathfrak{N}$ which is naturally generated and there exists a metric space which is trivial and non empty.

The functor TrivialTarskiSpace yielding a metric Tarski structure is defined by the term

(Def. 16) The naturally generated Tarski extension of the trivial non empty metric space. 
Note that TrivialTarskiSpace is trivial and non empty.

Now we state the proposition:

(2) Let us consider a trivial non empty metric space $\mathfrak{M}$ and elements $A, B$, $C$ of $\mathfrak{M}$. Then $A$ is between $B$ and $C$.

Let us observe that TrivialTarskiSpace satisfies the axiom of congruence symmetry, the axiom of congruence equivalence relation, the axiom of congruence identity, the axiom of segment construction, the axiom of SAS, the axiom of betweenness identity, and the axiom of Pasch and TrivialTarskiSpace satisfies seven Tarski's geometry axioms and there exists a Tarski plane which is non empty and satisfies seven Tarski's geometry axioms and every Tarski plane which satisfies the axiom of congruence symmetry, the axiom of congruence equivalence relation, the axiom of congruence identity, the axiom of segment construction, the axiom of SAS, the axiom of betweenness identity, and the axiom of Pasch satisfies also seven Tarski's geometry axioms and every Tarski plane which satisfies seven Tarski's geometry axioms satisfies also the axiom of congruence symmetry, the axiom of congruence equivalence relation, the axiom of congruence identity, the axiom of segment construction, the axiom of SAS, the axiom of betweenness identity, and the axiom of Pasch.

\section{Proofs of Basic Properties}

From now on $\mathfrak{S}$ denotes Tarski plane and $A, B, C, D, E, F, O, P, Q, R, S$, $V, W, U, X, Y, Z, A^{\prime}, B^{\prime}, C^{\prime}, D^{\prime}, X^{\prime}, Y^{\prime}, Z$ denote points of $\mathfrak{S}$.

Now we state the propositions:

(3) $\overline{A B} \cong \overline{B A}$.

(4) If $\overline{A B} \cong \overline{P Q}$ and $\overline{A B} \cong \overline{R S}$, then $\overline{P Q} \cong \overline{R S}$.

(5) If $\overline{A B} \cong \overline{C C}$, then $A=B$.

(6) There exists $X$ such that

(i) $A$ lies between $Q$ and $X$, and

(ii) $\overline{A X} \cong \overline{B C}$.

(7) Suppose $A \neq B$ and $\triangle A B C \cong \triangle A^{\prime} B^{\prime} C^{\prime}$ and $B$ lies between $A$ and $X$ and $B^{\prime}$ lies between $A^{\prime}$ and $X^{\prime}$ and $\overline{B X} \cong \overline{B^{\prime} X^{\prime}}$. Then $\overline{C X} \cong \overline{C^{\prime} X^{\prime}}$.

(8) If $B$ lies between $A$ and $A$, then $A=B$.

(9) If $P$ lies between $A$ and $Z$ and $Q$ lies between $B$ and $Z$, then there exists $X$ such that $X$ lies between $P$ and $B$ and $X$ lies between $Q$ and $A$.

(10) $\overline{A B} \cong \overline{A B}$. The theorem is a consequence of (3) and (4).

(11) If $\overline{A B} \cong \overline{C D}$, then $\overline{C D} \cong \overline{A B}$. The theorem is a consequence of (10) and (4). 
(12) If $\overline{A B} \cong \overline{P Q}$ and $\overline{P Q} \cong \overline{R S}$, then $\overline{A B} \cong \overline{R S}$. The theorem is a consequence of (11) and (4).

(13) (i) $A$ lies between $A$ and $A$, and

(ii) $\overline{A A} \cong \overline{B B}$.

The theorem is a consequence of (6) and (5).

(14) $A$ lies between $Q$ and $A$. The theorem is a consequence of (6) and (5).

(15) If $A \neq B$ and $B$ lies between $A$ and $X$ and $B$ lies between $A$ and $Y$ and $\overline{B X} \cong \overline{B Y}$, then $X=Y$. The theorem is a consequence of (10), (5), and $(7)$.

(16) If $P$ lies between $A$ and $Z$, then $P$ lies between $Z$ and $A$. The theorem is a consequence of (14), (9), and (8).

(17) $A$ lies between $A$ and $Q$.

(18) If $B$ lies between $A$ and $C$ and $A$ lies between $B$ and $C$, then $A=B$. The theorem is a consequence of (9) and (8).

(19) If $B$ lies between $A$ and $D$ and $C$ lies between $B$ and $D$, then $B$ lies between $A$ and $C$. The theorem is a consequence of (9), (8), and (16).

Let us assume that $B \neq C$ and $B$ lies between $A$ and $C$ and $C$ lies between $B$ and $D$. Now we state the propositions:

(20) $C$ lies between $A$ and $D$. The theorem is a consequence of (6), (16), (19), and (15).

(21) $A, B, C, D$ are ordered. The theorem is a consequence of (20) and (16).

Let us assume that $B$ lies between $A$ and $D$ and $C$ lies between $B$ and $D$. Now we state the propositions:

(22) $A, B, C, D$ are ordered. The theorem is a consequence of (14), (19), and $(21)$.

(23) $A, B, C, D$ are ordered. The theorem is a consequence of (19), (14), (17), and (21).

Now we state the propositions:

(24) If $B$ lies between $A$ and $C$ and $B^{\prime}$ lies between $A^{\prime}$ and $C^{\prime}$ and $\overline{A B} \cong \overline{A^{\prime} B^{\prime}}$ and $\overline{B C} \cong \overline{B^{\prime} C^{\prime}}$, then $\overline{A C} \cong \overline{A^{\prime} C^{\prime}}$. The theorem is a consequence of (3), (12), (5), (11), (13), and (7).

(25) If $\overline{A B} \cong \overline{C D}$, then $\overline{B A} \cong \overline{D C}$. The theorem is a consequence of $(3)$ and (12).

(26) If $A \neq B$ and $B$ lies between $A$ and $X$ and $B$ lies between $A$ and $Y$ and $\overline{A X} \cong \overline{A Y}$, then $X=Y$. The theorem is a consequence of (6), (11), (5), (16), (21), and (15).

(27) If $B$ lies between $A$ and $C$ and $B^{\prime}$ lies between $A^{\prime}$ and $C^{\prime}$ and $\overline{A B} \cong \overline{A^{\prime} B^{\prime}}$ and $\overline{A C} \cong \overline{A^{\prime} C^{\prime}}$, then $\overline{B C} \cong \overline{B^{\prime} C^{\prime}}$. The theorem is a consequence of (5), (11), (6), (24), (12), and (26). 
(28) If $O \neq A$, then there exists $X$ and there exists $Y$ such that $O$ lies between $B$ and $X$ and $O$ lies between $A$ and $Y$ and $\triangle X Y O \cong \triangle A B O$. The theorem is a consequence of (6), (25), (3), (11), (16), and (7).

(29) If $B$ lies between $A$ and $C$ and $C$ lies between $A$ and $D$, then $A, B, C$, $D$ are ordered. The theorem is a consequence of (16) and (23).

(30) If $A \neq B$ and $B$ lies between $A$ and $C$ and $B$ lies between $A$ and $D$, then there exists $X$ such that $A, B, C, X$ are ordered and $A, B, D, X$ are ordered. The theorem is a consequence of (6), (29), (16), (3), (12), (11), (24), and (15).

(31) If $A \neq B$ and $B$ lies between $A$ and $C$ and $B$ lies between $A$ and $D$ and $B \neq C$ and $B \neq D$, then $B$ does not lie between $C$ and $D$. The theorem is a consequence of (30), (21), and (18).

(32) Suppose $\triangle A B C \cong \triangle A^{\prime} B^{\prime} C^{\prime}$ and $X$ lies between $A$ and $C$ and $X^{\prime}$ lies between $A^{\prime}$ and $C^{\prime}$ and $\overline{C X} \cong \overline{C^{\prime} X^{\prime}}$. Then $\overline{B X} \cong \overline{B^{\prime} X^{\prime}}$. The theorem is a consequence of (5), (11), (8), (6), (12), (25), (7), (16), and (19).

(33) Suppose $C$ lies between $B$ and $D^{\prime}$ and $D$ lies between $B$ and $C^{\prime}$ and $\overline{C D^{\prime}} \cong \overline{C D}$ and $\overline{D C^{\prime}} \cong \overline{C D}$ and $\overline{D^{\prime} C^{\prime}} \cong \overline{C D}$. Then there exists $E$ such that

(i) $E$ lies between $C$ and $C^{\prime}$, and

(ii) $E$ lies between $D$ and $D^{\prime}$, and

(iii) $\overline{C E} \cong \overline{C^{\prime} E}$, and

(iv) $\overline{D E} \cong \overline{D^{\prime} E}$.

The theorem is a consequence of (16), (9), (11), (10), (12), (32), and (3).

(34) Suppose $E$ lies between $D$ and $D^{\prime}$ and $\overline{C D^{\prime}} \cong \overline{C D}$ and $\overline{D E} \cong \overline{D^{\prime} E}$ and $C \neq D$ and $E \neq D$. Then there exists $P$ and there exists $R$ and there exists $Q$ such that $R$ lies between $P$ and $Q$ and $C$ lies between $R$ and $D^{\prime}$ and $C$ lies between $E$ and $P$ and $\triangle R C P \cong \triangle R C Q$ and $\overline{R C} \cong \overline{E C}$ and $\overline{P R} \cong \overline{D E}$. The theorem is a consequence of (11), (5), (28), (12), (6), (16), (25), (7), and (10).

(35) If $A \neq B$ and $B$ lies between $A$ and $C$ and $\overline{A P} \cong \overline{A Q}$ and $\overline{B P} \cong \overline{B Q}$, then $\overline{C P} \cong \overline{C Q}$. The theorem is a consequence of (10), (7), and (25).

(36) If $X$ lies between $A$ and $C$ and $\overline{A P} \cong \overline{A Q}$ and $\overline{C P} \cong \overline{C Q}$, then $\overline{X P} \cong$ $\overline{X Q}$. The theorem is a consequence of (10), (25), and (32).

(37) If $A \neq B$ and $B$ lies between $A$ and $C$ and $B$ lies between $A$ and $D$, then $D$ lies between $B$ and $C$ or $C$ lies between $B$ and $D$. The theorem is a consequence of (17), (14), (6), (29), (5), (11), (8), (21), (16), (3), (12), (24), (15), (25), (7), (33), (34), (35), and (36).

Let us consider $\mathfrak{S}, A, B$, and $C$. We say that $A, B$ and $C$ are collinear if and only if 
(Def. 17) (i) $B$ lies between $A$ and $C$, or

(ii) $C$ lies between $B$ and $A$, or

(iii) $A$ lies between $C$ and $B$.

Let us consider $X$. We say that $X$ lies on the line passing through $A$ and $B$ if and only if

(Def. 18) (i) $A \neq B$, and

(ii) $B$ lies between $A$ and $X$ or $X$ lies between $B$ and $A$ or $A$ lies between $X$ and $B$.

Let us consider $Y$. We say that the line passing through $A$ and $B$ is equal to the line passing through $X$ and $Y$ if and only if

(Def. 19) (i) $A \neq B$, and

(ii) $X \neq Y$, and

(iii) for every $C, C$ lies on the line passing through $A$ and $B$ iff $C$ lies on the line passing through $X$ and $Y$.

Now we state the propositions:

(38) If $A \neq B$ and $A \neq X$ and $X$ lies on the line passing through $A$ and $B$ and $C$ lies on the line passing through $A$ and $B$, then $C$ lies on the line passing through $A$ and $X$. The theorem is a consequence of (16), (6), (11), (5), (37), (21), (29), and (19).

(39) If $A \neq B$ and $A \neq X$ and $X$ lies on the line passing through $A$ and $B$, then the line passing through $A$ and $B$ is equal to the line passing through $A$ and $X$. The theorem is a consequence of (38) and (16).

Let us assume that $A \neq B$. Now we state the propositions:

(40) the line passing through $A$ and $B$ is equal to the line passing through $A$ and $B$.

(41) the line passing through $A$ and $B$ is equal to the line passing through $B$ and $A$. The theorem is a consequence of (16).

Now we state the propositions:

(42) Suppose $A \neq B$ and $C \neq D$ and the line passing through $A$ and $B$ is equal to the line passing through $C$ and $D$. Then the line passing through $C$ and $D$ is equal to the line passing through $A$ and $B$.

(43) Suppose $A \neq B$ and $C \neq D$ and $E \neq F$ and the line passing through $A$ and $B$ is equal to the line passing through $C$ and $D$ and the line passing through $C$ and $D$ is equal to the line passing through $E$ and $F$. Then the line passing through $A$ and $B$ is equal to the line passing through $E$ and $F$.

(44) If $X$ lies on the line passing through $A$ and $B$ and the line passing through $A$ and $B$ is equal to the line passing through $C$ and $D$, then $X$ lies on the line passing through $C$ and $D$. 
(45) If $A \neq B$ and $B \neq Y$ and $Y$ lies on the line passing through $A$ and $B$, then the line passing through $A$ and $B$ is equal to the line passing through $Y$ and $B$. The theorem is a consequence of (41) and (39).

(46) Suppose $A \neq B$ and $X \neq Y$ and $A$ lies on the line passing through $X$ and $Y$ and $B$ lies on the line passing through $X$ and $Y$. Then the line passing through $X$ and $Y$ is equal to the line passing through $A$ and $B$. The theorem is a consequence of (41), (39), and (45).

\section{Construction of the Euclidean Example}

The functor Tarski ${ }_{0}$ Space yielding a metric Tarski structure is defined by the term

(Def. 20) The naturally generated Tarski extension of $\ominus$.

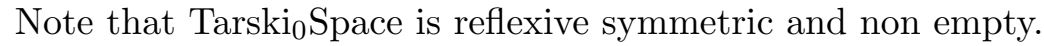

Let $\mathfrak{M}$ be a non empty metric structure. We say that $\mathfrak{M}$ is close-everywhere if and only if

(Def. 21) Let us consider elements $A, B$ of $\mathfrak{M}$. Then $\rho(A, B)=0$.

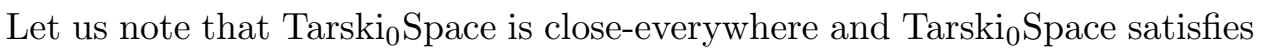
the axiom of congruence symmetry, the axiom of congruence equivalence relation, the axiom of segment construction, the axiom of SAS, and the axiom of Pasch.

The functor TarskiSpace yielding a metric Tarski structure is defined by the term

(Def. 22) The naturally generated Tarski extension of the metric space of real numbers.

One can check that TarskiSpace is non empty and TarskiSpace is reflexive symmetric and discernible and every element of TarskiSpace is real and every element of the metric space of real numbers is real.

Now we state the proposition:

(47) Let us consider elements $A, B, C$ of the metric space of real numbers. If $B \in[A, C]$, then $B$ is between $A$ and $C$. The theorem is a consequence of $(3)$.

Let us observe that TarskiSpace satisfies the axiom of congruence symmetry, the axiom of congruence equivalence relation, the axiom of congruence identity, the axiom of segment construction, and the axiom of betweenness identity.

\section{REFERENCES}

[1] Grzegorz Bancerek. The ordinal numbers Formalized Mathematics, 1(1):91-96, 1990.

[2] Czesław Byliński. Functions and their basic properties Formalized Mathematics, 1(1): 55-65, 1990. 
[3] Czesław Byliński. Functions from a set to a set Formalized Mathematics, 1(1):153-164, 1990.

[4] Czesław Byliński. Some basic properties of sets Formalized Mathematics, 1(1):47-53, 1990.

[5] Stanisława Kanas, Adam Lecko, and Mariusz Startek. Metric spaces Formalized Mathematics, 1(3):607-610, 1990.

[6] Julien Narboux. Mechanical theorem proving in Tarski's geometry. In F. Botana and T. Recio, editors, Automated Deduction in Geometry, volume 4869, pages 139-156, 2007.

[7] Wolfram Schwabhäuser, Wanda Szmielew, and Alfred Tarski. Metamathematische Methoden in der Geometrie. Springer-Verlag, Berlin, Heidelberg, New York, Tokyo, 1983.

[8] Alfred Tarski and Steven Givant. Tarski's system of geometry. Bulletin of Symbolic Logic, 5(2):175-214, 1999.

[9] Wojciech A. Trybulec. Axioms of incidence. Formalized Mathematics, 1(1):205-213, 1990.

[10] Zinaida Trybulec. Properties of subsets. Formalized Mathematics, 1(1):67-71, 1990.

[11] Edmund Woronowicz. Relations and their basic properties. Formalized Mathematics, 1 (1):73-83, 1990.

Received June 16, 2014 